\title{
CLASSICAL AND QUANTUM INTERACTION OF THE DIPOLE
}

\author{
J. Anandan \\ Department of Physics and Astronomy, University of South Carolina, \\ Columbia, SC 29208. \\ E-mail: jeeva@sc.edu
}

(Oct. 4, 99)

\begin{abstract}
A unified and fully relativistic treatment of the interaction of the electric and magnetic dipole moments of a particle with the electromagnetic field is given. New forces on the particle due to the combined effect of electric and magnetic dipoles are obtained. Four new experiments are proposed, three of which would observe topological phase shifts.
\end{abstract}

A beautiful aspect of electromagnetism, which is becoming increasingly fundamental, is the duality between electric and magnetic fields. It is therefore natural to extend the many interesting facets of the influence of the electromagnetic field on the magnetic dipole [1-7] to the dual situations [8.9]. In this letter, I shall give a model independent and fully relativistic derivation of the low energy Lagrangian for the interaction of the electric and magnetic dipoles with an external electromagnetic field [10]. This treatment applies to elementary particles, nuclei, atoms and molecules that have an electric or magnetic dipole or both. New forces on the dipole will be obtained using the non abelian nature of the interaction [4 [6]. I shall then propose new feasible experiments to test this interaction, three of which would have topological aspects analogous to the Aharonov-Bohm (AB) effect [11].

The simplest classical Lorentz invariant action for a particle of mass $m$ interacting with the electromagnetic field strength $F_{\mu \nu}$ is

$$
I=-\int m c d s-\frac{1}{2 c} \int F_{\mu \nu} D^{\mu \nu} d s
$$


where $s$ is the proper time along the particle's world- line. For simplicity the total electric and magnetic charges of the particle have been assumed to be zero. $D^{\mu \nu}$ may be taken to be anti-symmetric without loss of generality, and will be called the dipole moment tensor. More generally, $I=\int \mathcal{L} d s$, where $\mathcal{L}$ is a Lorentz scalar that is an analytic function of $F_{\mu \nu}$. In the power series expansion of $\mathcal{L}$ in $F_{\mu \nu}$, the sum of the first and higher order terms is the last term in (11). In the corresponding power series for $D^{\mu \nu}$, the term that is independent of $F_{\mu \nu}$ is called the intrinsic dipole moment and the remainder is called the induced dipole moment.

In any frame, $D^{0 i}$ and $D^{i j}$, that couple, respectively, to the electric field components $F_{0 i}$ and the magnetic field components $F_{i j}$, are called the components of the electric and magnetic dipole moments. In the rest frame of the particle, $D^{0 i}$ and $D^{i j}$ represent the intrinsic electric and magnetic dipole moments, much like the energy in the rest frame representing the mass of the particle. These may be covariantly represented, respectively, by the electric and magnetic dipole moment 4-vectors

$$
d^{\mu} \equiv D_{\nu}^{\mu} v^{\nu}, \quad m^{\mu} \equiv \frac{1}{2} \epsilon^{\mu \nu \rho \sigma} D_{\nu \rho} v_{\sigma}
$$

where the 4-velocity $v^{\mu}=\frac{d x^{\mu}}{d s}$. Then

$$
d_{\mu} v^{\mu}=0, \quad m_{\mu} v^{\mu}=0
$$

This reflects the fact that only three components of each dipole moment 4 -vector are independent, because in the rest frame, in which $v^{\mu}=(1,0,0,0)$, each vector has only three (spatial) components.

Now the identity $\epsilon^{\mu \nu \rho \sigma} \epsilon_{\mu \alpha \beta \gamma}=-6 \delta_{[\alpha}^{\nu} \delta_{\beta}^{\rho} \delta_{\gamma]}^{\sigma}$, implies [12]

$$
\frac{1}{2} F_{\mu \nu} D^{\mu \nu}=d^{\mu} F_{\mu \nu} v^{\nu}+m^{\mu} F *_{\mu \nu} v^{\nu}
$$

The electric and magnetic fields in the rest frame may also be covariantly defined by

$$
E^{\mu}=F_{\nu}^{\mu} v^{\nu}, \quad B^{\mu}=\frac{1}{2} \epsilon^{\mu \nu \rho \sigma} F_{\nu \rho} v_{\sigma}=F *^{\mu}{ }_{\nu} v^{\nu}
$$

Then $E^{\mu} v_{\mu}=0=B^{\mu} v_{\mu}$. In the rest frame, $E^{\mu}=(0, \mathbf{E})$ and $B^{\mu}=(0, \mathbf{B})$, where $\mathbf{E}$ and $\mathbf{B}$ are the electric and magnetic (spatial) vectors, in the usual notation. On using (5), (田) reads 


$$
\frac{1}{2} F_{\mu \nu} D^{\mu \nu}=d^{\mu} E_{\mu}+m^{\mu} B_{\mu}
$$

On defining the 4-vector potentials,

$$
D_{\nu}=d^{\mu} F_{\mu \nu}, \quad M_{\nu}=m^{\mu} F *_{\mu \nu}
$$

(匹) may also be rewritten as

$$
\frac{1}{2} F_{\mu \nu} D^{\mu \nu}=D_{\mu} v^{\mu}+M_{\mu} v^{\mu}=a_{\mu} v^{\mu}
$$

where $a_{\mu}=D_{\mu}+M_{\mu}$.

From (11) and (8), the relativistic lagrangian is

$$
L_{R}=-m c \frac{d s}{d t}-M_{\mu} \frac{d x^{\mu}}{d t}-D_{\mu} \frac{d x^{\mu}}{d t}
$$

Clearly then, the duality between the interactions of the electric and magnetic dipoles is complete to all orders of $v / c$ indepenently whether or not there are magnetic monopoles. Each of the last two terms in (9) is analogous to the electromagnetic interaction term $e A_{\mu} \frac{d x^{\mu}}{d t}$ in the Lagrangian for a charged particle, where $A_{\mu}$ is the electromagnetic 4-vector potential. This immediately suggests topological effects analogous to the AB effect [11].

When the Lagrangian is quantized, the phase shift that the particle experiences due to the the electromagnetic field, using the action (1), and (8), is given by the phase factor

$$
P \exp \left(-\frac{i}{2 c \hbar} \int_{\gamma} F_{\mu \nu} D^{\mu \nu} d s\right)=P \exp \left(-\frac{i}{\hbar c} \int_{\gamma} a_{\mu} d x^{\mu}\right)
$$

where $\mathrm{P}$ represents path ordering because $D^{\mu \nu}, D_{\mu}, M_{\mu}$ and therefore $a_{\mu}$ are now operators which need not commute. For a topological phase, the effect of (10) on the wave function should not change when $\gamma$ is deformed in a suitable region. Such a deformation may be obtained by infinitesimal deformations. To see the effect of an infinitesimal deformation, consider (10) around an infinitesimal closed curve spanning a surface element $d \sigma^{\mu \nu}$ :

$$
P \exp \left(-\frac{i}{\hbar c} \oint_{\gamma} a_{\mu} d x^{\mu}\right)=1-\frac{i}{2 \hbar c} G_{\mu \nu} d \sigma^{\mu \nu}
$$

where the "Yang-Mills field strength" $G_{\mu \nu}$ is defined by

$$
G_{\mu \nu}=\partial_{\mu} a_{\nu}-\partial_{\nu} a_{\mu}-\frac{i}{\hbar c}\left[a_{\mu}, a_{\nu}\right]
$$


An external field modifies the motion of a wave function, via Huyghen's principle, by the phase shift that it causes in the interference of secondary wavelets. This gives a relativistic correspondence principle that enables the classical equation of motion to be determined from the phase shift around an infinitesimal closed curve [13]. Let $\xi^{\mu}(t)=\left(t,<\psi(t)\left|x^{i}\right| \psi(t)>\right)$ represent the world-line of a wave-packet $\mid \psi(t)>$ of the particle, where $x^{i}$ are the position operators. Then for a suitably localized wave-packet, the 4 -force

$$
f^{\mu} \equiv m \frac{d^{2} \xi^{\mu}}{d s^{2}}=<\psi\left|G^{\mu}{ }_{\nu}\right| \psi>\frac{d \xi^{\mu}}{d s}
$$

to a good approximation. The approximation to (13) in the low energy limit, to $O(1 / c)$, was obtained by writing $d<x^{i}>/ d t$ and $d^{2}<x^{i}>/ d t^{2}$ in terms of the commutators of the Hamiltonian [5]. Then Lorentz covariance implies that (13) is valid to all orders in $1 / c$. A purely classical derivation of (13), with the expectation values replaced by the corresponding classical quantities, may be obtained by replacing the above quantum commutators by appropriate Poisson brackets of the relativistic classical Hamiltonian. The 4-force (13) is also analogous to the 4-force on a Yang-Mills particle obtained in the classical limit of the Dirac's equation by Wong [14].

(11) and (13) show that $G_{\mu \nu}$ determines both whether the phase shift is topological (i.e. whether (10) is unchanged when $\gamma$ is varied) and non local (the force on the interfering beams is zero). If

$$
G_{\mu \nu}=0
$$

everywhere in a non simply connected region to which the interfering beams are confined, then the phase shift will be called strongly topological or non local. The phase shift will be called weakly topological or non Local if

$$
<\psi\left|G_{\mu \nu}\right| \psi>=0
$$

It is interesting that the last term in (11), which appears to be a non gauge interaction, may be regarded as a gauge interaction in the fully relativistic theory because of the identity (8). Hence, the topological and geometrical phases implied by the Lagrangian (9) are also valid to all orders in $1 / c$, unlike in earlier treatments. I shall now make a low energy 
approximation by neglecting terms of $O\left(1 / c^{2}\right)$. From (7) and (9), the parts of $D_{\mu}$ and $M_{\mu}$ that contribute to $L_{R}$ in this approximation are

$$
\begin{aligned}
D_{\mu}= & (-\mathbf{d} \cdot \mathbf{E}, \mathbf{d} \times \mathbf{B}), M_{\mu}=(-\mu \cdot \mathbf{B},-\mu \times \mathbf{E}), \\
& a_{\mu}=M_{\mu}+D_{\mu}
\end{aligned}
$$

The Lagrangian obtained in this approximation from (9), after subtracting the rest mass energy, is [15]

$$
L=\frac{1}{2} m v^{2}+\mu \cdot \mathbf{B}+\mathbf{v} \cdot \mu \times \mathbf{E}+\mathbf{d} \cdot \mathbf{E}-\mathbf{v} \cdot \mathbf{d} \times \mathbf{B}
$$

Then the Hamiltonian, obtained by the usual Legendre transformation from this Lagrangian, is

$$
\begin{aligned}
H= & \frac{1}{2 m}(\mathbf{p}-\mu \times \mathbf{E}+\mathbf{d} \times \mathbf{B})^{2}-\mu \cdot \mathbf{B}-\mathbf{d} \cdot \mathbf{E} \\
& \equiv \frac{1}{2 m}(\mathbf{p}-\mathbf{a})^{2}+a_{0}
\end{aligned}
$$

The velocity of the center of the wave packet is

$$
\begin{aligned}
v^{i} \equiv & \frac{d}{d t}<\psi\left|x^{i}\right| \psi>=\frac{i}{\hbar}<\psi\left|\left[H, x^{i}\right]\right| \psi> \\
& =\frac{1}{m}<\psi\left|p^{i}-a^{i}\right| \psi>
\end{aligned}
$$

The force is

$$
m \frac{d v^{i}}{d t}=\frac{i}{\hbar}<\psi\left|\left[H, p^{i}-a^{i}\right]\right| \psi>
$$

This gives the force to be the same as the spatial components of (13), in the present limit.

Suppose now that the dipole is made of two particles with charges $q,-q$, masses $m_{1}, m_{2}$ and coordinates $\mathbf{x}_{1}, \mathbf{x}_{2}$. It can be shown that the oribital angular momentum of the system about an arbitrary origin

$$
\mathbf{x}_{1} \times \mathbf{p}_{1}+\mathbf{x}_{2} \times \mathbf{p}_{2}=\mathbf{x} \times\left(\mathbf{p}_{1}+\mathbf{p}_{2}\right)+\mathbf{r} \times \mathbf{p}
$$

where $\mathbf{x}=\left(m_{1}+m_{2}\right)^{-1}\left(m_{1} \mathbf{x}_{1}+m_{2} \mathbf{x}_{2}\right)$ is the center of mass, $\mathbf{r}=\mathbf{x}_{1}-\mathbf{x}_{2}$ the relative coordinates, and 


$$
\mathbf{p}=\frac{1}{m_{1}+m_{2}}\left(m_{2} \mathbf{p}_{1}-m_{1} \mathbf{p}_{2}\right)
$$

Then, $\left[p_{i}, r_{j}\right]=-i \hbar \delta_{i j}$ and $\left[p_{i}, x_{j}\right]=0$. The last term in (21), $\mathbf{L} \equiv \mathbf{r} \times \mathbf{p}$, is the total orbital angular momentum about the center of mass, and is invariant under Galilei boosts. It satisfies

$$
\left[L_{i}, L_{j}\right]=i \hbar \epsilon_{i j k} L_{k},\left[L_{i}, r_{j}\right]=i \hbar \epsilon_{i j k} r_{k},\left[L_{i}, x_{j}\right]=0
$$

In general, the magnetic moment

$$
\mu=\gamma_{L} \mathbf{L}+\gamma_{S} \mathbf{S}
$$

where $\mathbf{S}$ is the total spin. The electric dipole moment $\mathbf{d}=q \mathbf{r}$. It follows that

$$
\begin{gathered}
{\left[\mu_{i}, \mu_{j}\right]=i \hbar \epsilon_{i j k}\left(\gamma_{L}^{2} L_{k}+\gamma_{S}^{2} S_{k}\right),\left[d_{i}, d_{j}\right]=0} \\
{\left[\mu_{i}, d_{j}\right]=i \hbar \gamma_{L} \epsilon_{i j k} d_{k}}
\end{gathered}
$$

In the rest frame of the dipole, i.e. a frame in which (19) is zero, from (13), $f^{0}=0$ and $f^{i}=<\psi\left|G_{0}^{i}\right| \psi>$. In the present low energy limit, the latter force is

$$
\begin{aligned}
\mathbf{f}= & \nabla(\mu \cdot \mathbf{B}+\mathbf{d} \cdot \mathbf{E})+\frac{1}{c} \frac{\partial}{\partial t}(\mathbf{d} \times \mathbf{B}-\mu \times \mathbf{E}) \\
& -\frac{\gamma_{S}^{2}}{c}(\mathbf{S} \times \mathbf{B}) \times \mathbf{E}-\frac{\gamma_{L}^{2}}{c}(\mathbf{L} \times \mathbf{B}) \times \mathbf{E} \\
& -\frac{\gamma_{L}}{c}(\mathbf{B} \times \mathbf{d}) \times \mathbf{B}-\frac{\gamma_{L}}{c}(\mathbf{E} \times \mathbf{d}) \times \mathbf{E}
\end{aligned}
$$

(25) may also be obtained from (20). $\mu, \mathbf{d} . \mathbf{S}$ and $\mathbf{L}$ in (25), quantum mechanically, are expectation values of the corresponding operators. The force in an arbitrary inertial frame may be obtained by Lorentz transforming the above $f^{\mu}$ to this frame. Of the terms in (25) that are non linear in the electromagnetic field, $-\left(\gamma_{S}^{2} / c\right)(\mathbf{S} \times \mathbf{B}) \times \mathbf{E}$ was previously obtained by the author [5,6] and may be experimentally detectable [16]. The remaining three non linear terms are new. Particularly interesting are the last two terms in (25), which are due to the combined effect of the electric and magnetic dipole moments.

An experiment that would detect a topological effect due to $D_{0}$, which is analogous to the scalar AB effect [11], is the following. Split a beam of identical neutral particles with electric dipole moment $\mathbf{d}$, but no magnetic dipole moment, into two, and send one beam between a pair of capacitor plates that are initially neutral. The beam is so weak that at 
most one particle is inside the capacitor at any given time. The beam is also polarized so that $\mathbf{d}$ is parallel to the electric field in the capacitor. When each particle is inside the capacitor turn on the homogeneous electric field of the capacitor and turn it off before the particle leaves the capacitor plate. Then the phase shift due to $D_{0}$ is [18]

$$
-\frac{1}{\hbar} \int_{0}^{T} D_{0} d t=\frac{1}{\hbar} \int_{0}^{T} \mathbf{d} \cdot \mathbf{E} d t=(E T d / \hbar)
$$

This is analogous to the experiment to detect the topological phase shift due to $M_{0}$ proposed by Zeilinger [17] in analogy with the scalar AB effect [11], and by the author [6] as the scalar phase shift corresponding to the vector phase shift considered by Aharonov and Casher [2] in the interference of magnetic moment around a line charge. This scalar phase shift has been experimentally observed [19,20].

In the above mentioned three interferometry experiments with electric or magnetic dipoles, it is the expectation value of the quantum force operator, or the classical force, that vanishes, whereas the quantum force, $m\left(d^{2} \hat{x}^{i} / d t^{2}\right)$, is non zero [21]. For example, in the second of the above three experiments [17,6], the linear terms in the quantum force vanish because of the homogeneity of the magnetic field, on using a Maxwell's equation. But the non linear term $-\gamma_{S}^{2}(\hat{\mathbf{S}} \times \mathbf{B}) \times \mathbf{E}$ does not vanish. It is the expectation value of the latter term, or the corresponding classical force term, that vanishes. All three experiments are weakly topological because, along the interfering beams, (15) holds whereas (14) does not hold. This is analogous to the Stern-Gerlach experiment with the spin perpendicular to the inhomogeneity of the magnetic field for which also (15) holds whereas (14) does not hold. In the latter experiment the existence of the quantum force is obvious because it splits the beam into two, even though the classical force is zero. On the other hand, the AB effect 11] is strongly topological, as defined above, because $G_{\mu \nu}=e F_{\mu \nu}=0$ in this case.

However, in the following proposed interferometric experiments (14) holds, and hence the quantum force is zero and the phase shifts are strongly topological and non local. Subject the entire interferometer to a homogeneous electric or magnetic field. Along each beam the magnetic or electric dipole moment is rotated by $180^{\circ}$ and is rotated back again by $180^{\circ}$ by suitable oscillating fields after the beams have traveled distances $L_{1}$ and $L_{2}$, respectively. The contributions to the phase shift due to the dipole flips on one beam is canceled by the ones in the other beam by doing them identically. By varying $L_{1}-L_{2}$ on which the phase 
shift depends, it can be shown clearly that the phase shift is due to the quantum force free interaction of the dipole. In a neutron interference experiment, for example, if the entire interferometer subject to a homogeneous electric field $\mathbf{E}$, the phase shifts for neutron spin components parallel and anti-parallel to $\mathbf{E} \times \mathbf{v}$ are [1]

$$
\pm \frac{2|\mathbf{M}|}{\hbar}\left(L_{1}-L_{2}\right)= \pm \frac{2 \mu E}{\hbar}\left(L_{1}-L_{2}\right) .
$$

The most sensitive method for detecting the electric dipole interaction is by the electric resonance method described by Ramsey [22]. Here a polarized molecular beam is deflected and refocused by two inhomogeneous electric fields. In between, a homogeneous and oscillatory electric fields are applied. The fields are chosen so that an allowed electric dipole transition occurs due to the latter fields which makes the intensity of the refocused beam maximum. Introduce a magnetic field $\mathbf{B}$ in the intermediate region so that $\mathbf{v} \times \mathbf{B}$ is in the direction of the homogeneous electric field. This shifts the intensity from the maximum which may easily be detected.

I thank Kuniharu Kubodera and Norman F. Ramsey for useful discussions. 


\section{REFERENCES}

[1] J. Anandan, Phys. Rev. Lett. 24, 1660 (1982).

[2] Y. Aharonov and A. Casher, Phys. Rev. Lett. 53, 319 (1984).

[3] Y. Aharonov, P. Pearle and L. Vaidman, Phys. Rev. A 37, 4052 (1988).

[4] A. S. Goldhaber, Phys. Rev. Lett. 62, 380 (1989).

[5] J. Anandan, Phys. Lett. A 138, 347 (1989), errata: Phys. Lett. 152A, 504 (1991).

[6] J. Anandan in Proceedings of the International symposium on the Foundations of Quantum Mechanics, Tokyo, August 1989, edited by S. Kobayashi et al (Physical Society of Japan, 1990) p. 98.

[7] Norman F. Ramsey, Phys. Rev. A 48, 80 (1993).

[8] M. Wilkens, Phys. Rev. Lett. 72, 5 (1994).

[9] G. Spavieri , Phys. Rev. Lett. 81, 1533 (1998); H. Wilkens, Phys. Rev. Lett. 81, 1534 (1998); G. Spavieri, Phys. Rev. A 59, 3194 (1999).

[10] Such a model independent derivation seems necessary because the electric dipole, which is usually thought of as due to separated opposite electric charges considered later in this leter, may be due to other distributions, e.g. differential rotation of magnetic charges, if they exist, as pointed out by E. M. Purcell and N.F. Ramsey, Phys. Rev. 807 (1950).

[11] Y. Aharonov and D. Bohm, Phys. Rev. 115, 485 (1959).

[12] The dual electromagnetic field $F *_{\mu \nu} \equiv \frac{1}{2} \epsilon_{\mu \nu \rho \sigma} F^{\rho \sigma}$ where $\epsilon_{\mu \nu \rho \sigma}$ is the volume form that is the completely anti- symmetric tensor with $\epsilon_{0123}=1$ in every inertial frame.

[13] J. Anandan, Phys. Rev. D, 15, 1448 (1977), sec. 4; J. Anandan, Int. J. Theor. 19, 537 (1980).

[14] S. K. Wong, Nuov. Cim. A A65, 689 (1970). See also R. Kerner, Ann. Inst. Henri poincare 9, 143 (1968); A. P. Balachandran, S. Borchardt, and A. Stern, Phys. Rev. D, 17, 3247 (1978). 
[15] The $\mathbf{v} \cdot \mu \times \mathbf{E}$ and $\mathbf{v} \cdot \mathbf{d} \times \mathbf{B}$ terms were obtained previously in [5] and [8], respectively, from the interactions of the dipoles in the rest frame, even though the Lagrangian is written in the laboratory frame. But both these heuristic arguments may be justified using (6) of the present letter.

[16] A. Wagh and V. C. Rakhecha, Phys. Rev. Lett., 78, 1399-1403 (1997); J. Anandan, Nature, 387, 558-559 (5 June 1997).

[17] A. Zeilinger in Fundamental Aspects of Quantum Theroy, edited by V. Gorini and A. Frigerio (Plenum, NY 1985).

[18] In the experiment proposed to detect the phase shift of $D_{0}$ in ref [8], two different fields are applied to the two arms of the interferometer. However, in the regions where the electric field changes spatially, the beam would experience a force. It is necessary to make the electric field time dependent and the beam sufficiently weak as in the present letter in order for the phase shift to be topological and non local.

[19] G. Badurek, H. Weinfurter, R. Gähler, A. Kollmar, S. Wehinger and A. Zeilinger, Phys. Rev. Lett. 71, 307 (1993). This experiment shows that this phase is non dispersive, i.e. independent of the wavelength. This is important in establishing its geometrical nature.

[20] B. E. Allman et al, Phys. Rev. Lett. 68, 2409 (1992), errata: Phys. Rev. Lett. 70, 250 (1993); Phys. Rev. A 48, 1799 (1993).

[21] J. Anandan and C. R. Hagen, Phys. Rev. A 50, 2860- 2864 (1994), footnote 9.

[22] Norman F. Ramsey, Molecular Beams (Oxford Univ. Press, 1990), p. 298-301. 